\title{
A comparative analysis of foundation design solutions on permafrost soils
}

\author{
Ivan Inzhutov ${ }^{1}$, Victor Zhadanov ${ }^{2}$, Maxim Semenov ${ }^{1}$, Sergei Amelchugov ${ }^{1}$, Alexey \\ Klimov ${ }^{1}$, Peter Melnikov ${ }^{1}$, and Nadezhda Klinduh ${ }^{1, *}$ \\ ${ }^{1}$ Siberian Federal University, Pr. Svobodny, 82, Krasnoyarsk, 660041, Russia \\ ${ }^{2}$ Orenburg State University, Pr. Pobedy, 13, Orenburg, 460018, Russia
}

\begin{abstract}
The article analyzes design solutions of foundations on permafrost soils, their advantages and disadvantages. Innovative design solutions that increase the energy efficiency of buildings are proposed. Examples of innovative foundations of platform type are given. Spatial ventilated foundations are less sensitive to soil deformation. The construction of such a foundation can be made of various materials such as reinforced concrete, metal and timber. A spatial foundation platform based on timber is proposed as one of the innovative examples, which is a promising constructive solution of foundations for construction in the Arctic regions. Wood has a small coefficient of thermal conductivity, which significantly increases the energy efficiency of the structure as a whole. Due to prefabrication of timber structures, the speed of construction is increased. Platforms can have solutions in the form of: system of cross beams, structural plates, plate-rod structure, as well as in the form of shells and folds. Regardless of the design solution, the spatial foundation platform is prefabricated.
\end{abstract}

\section{Introduction}

Construction of buildings and structures on permafrost soils is relevant, problematic and at the same time promising for the countries located in the Northern latitudes. The subsoil in permafrost hides a lot of minerals that dictates the need to develop the Arctic territory. If two thirds of the territory of the Russian Federation is frozen soils, then the countries of Northern Europe are forced to live in permafrost. For living and mastering the Arctic zone, it is necessary to erect temporary and permanent buildings and structures on permafrost soils [1].

There are two principles of using permafrost soils as the foundations of structures. Construction according to the first principle requires special design solutions aimed at preserving the soil in a frozen state, since thawing of the soil will lead to critical deformations. The construction of foundations on permafrost soils on the second principle is not particularly different from the installation of the foundation on thawed soils. The footing is a low compressible soil or soil deformation during thawing does not exceed the maximum permissible values for the construction.

\footnotetext{
* Corresponding author: NKlindukh@sfu-kras.ru
} 
For example, a ventilated underground is an example of the use of the footing of the building on the I principle. The presence of a ventilated underground is possible with various structural solutions of foundations and the structure itself. Space ventilation between the overlap of the heated floor and the footing helps to prevent the transfer of heat to the frozen ground.

\section{Materials and Methods}

There exists experience of construction of pile foundations on permafrost. as discussed by Goncharov. Based on the analysis of existing foundations in the city of Norilsk, bored piles located in the space of ventilated underground are the mostly used ones (Fig. 1).

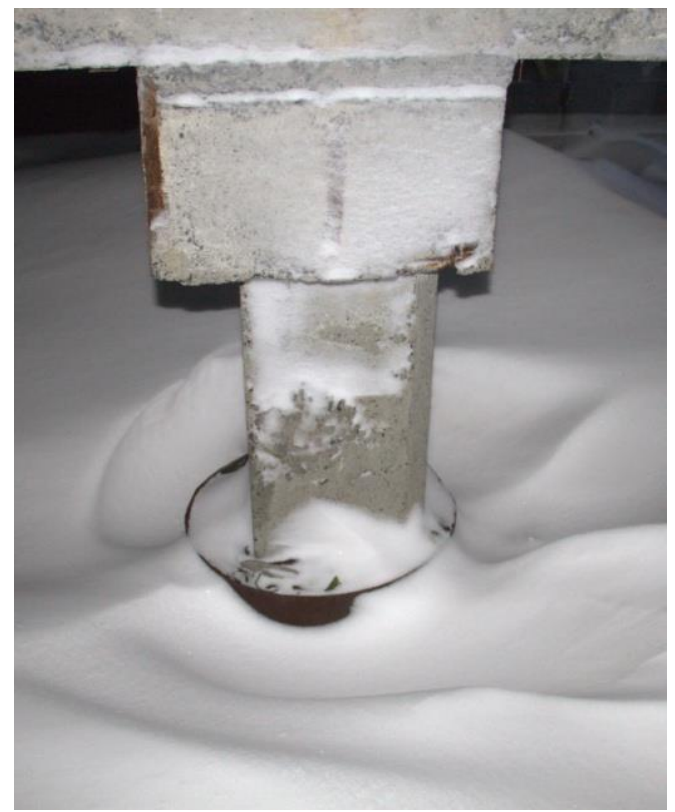

Fig. 1. Boring pile (case of Norilsk).

Taking into account the complex geological structure of the soil, during the installation casing metal pipes were used (Fig. 2). 


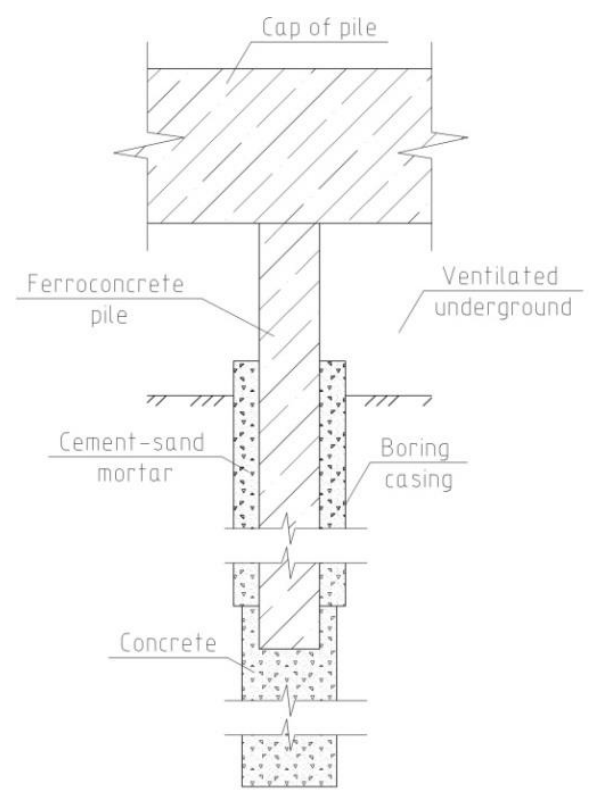

Fig. 2. Scheme of boring pile (case of Norilsk).

Norilsk is largely built on bulk soils, under layers of which peat and highly compressible soils are hidden. According to the authors, the degradation of frozen soil of Norilsk is caused by a number of reasons. Along with global warming and man-made impact on the soil, there is also a constructive reason. The presence of heat-conducting materials, which are part of the pile structure, promotes the splitting of the soil, which leads to its deformations.

When installing temporary buildings and structures, screw pile technology is common. The absence of earthworks and the ability to dismantle piles make this technology less labor-intensive. There is a technology in which a steel or reinforced concrete pipe is inserted into a pre-drilled well. Such a pipe is called a shell pile (Fig. 3).

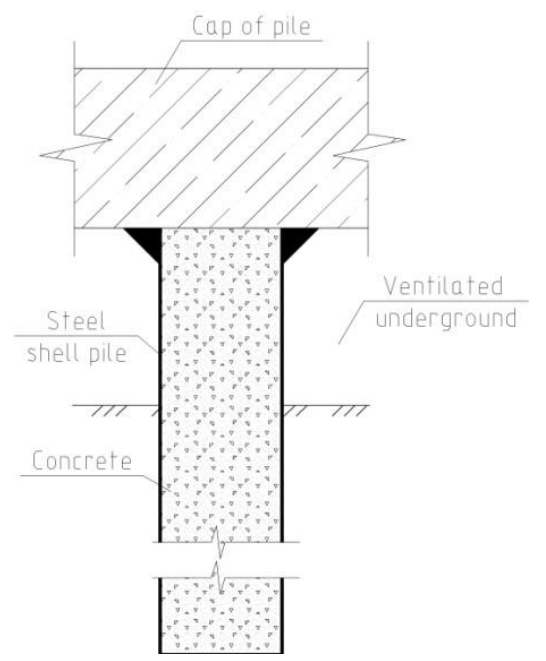

Fig. 3. Scheme of steel shell pile 
The tube body is filled in with concrete, but soil is in certain cases used. Then the well freezes and the soil temperature is controlled by special sensors. Accommodation facilities, equipment and pipelines in oil-producing areas are mainly built with the help of screw piles and steel shell pile (Fig. 4).

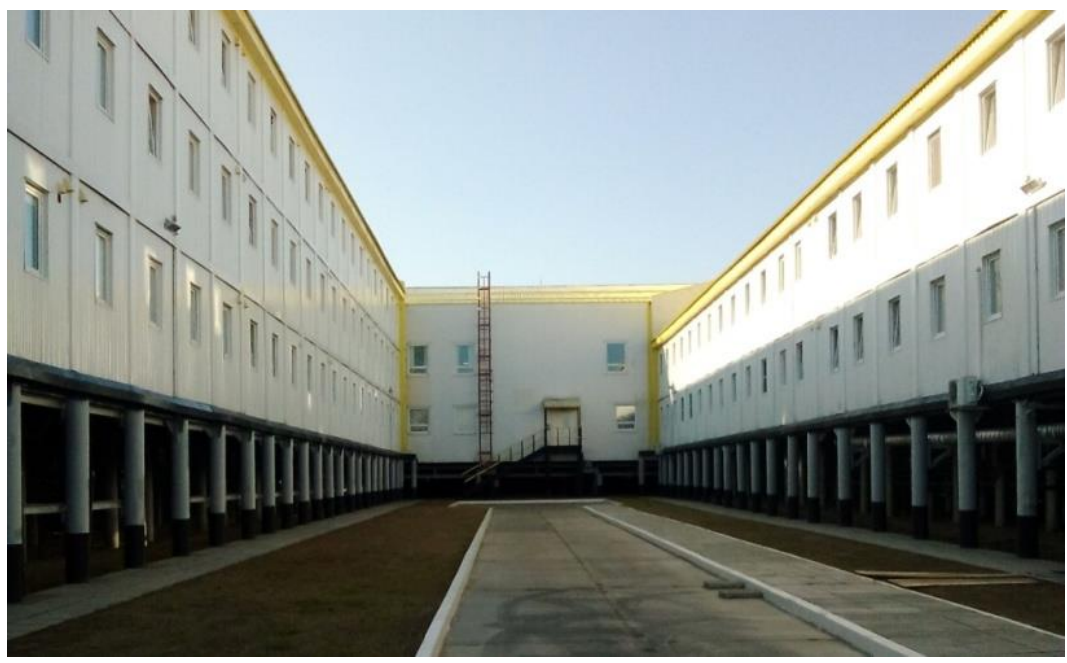

Fig. 4. Structures erected on steel shell pile

In addition to point foundations there is global experience of designing and building spatial foundations which have properly established themselves. These include the spatial foundations of the shell and the spatial foundations of the structural type. The developed spatial foundations are ventilated (Fig. 5). Such foundations made of reinforced concrete were built on the territory of Yakutia, Igarka and the Norilsk Oganer industrial district according to Goncharov.

Also known technology Multipoint Foundations widely used in the United States and Canada. Multipoint Foundations is ready-made and erected from metal elements (Fig. 5).

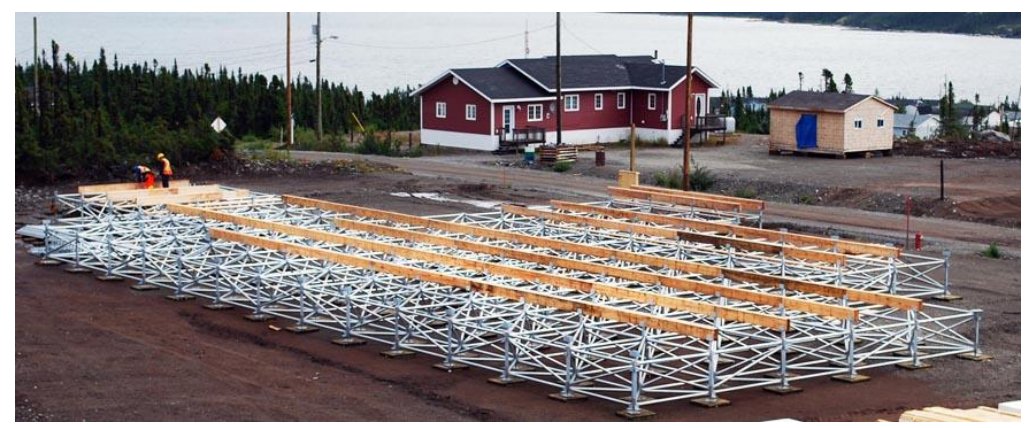

Fig. 5. Structures erected on steel shell pile, Source: http://multipoint-foundations.com

According to the authors, spatial ventilated foundations are less sensitive to soil deformation. The construction of such a foundation can be made of various materials such as reinforced concrete, metal and timber.

There exists the Abovsky spatial foundation platform. The platform consists of a lower and upper belt of structural elements. The lower belt is installed on the outer surface of the soil. Upper and lower belts are assembled from precast concrete elements. The upper and lower belts are interconnected by metallic spatial strut - framed beam. The use of spatial 
foundation platforms is promising for a number of reasons as discussed by Abovsky and Mitasov:

- reduction of engineering-geological surveys;

- the structure does not require a large number of excavation works, which in the case of frozen soils is quite time-consuming;

- the spatial foundation platform is less sensitive to the deformation of the footing soil due to the integral work of the structure, it is advisable to erect them in seismically active areas, on weak and structurally unstable soils, including permafrost soils;

- the possibility of the structure of a spatial foundation platform to be adjustable, for example, by means of jack devices;

- availability of repair and reinforcement of the foundation structure;

- platform prefabrication solves the issues of transportation and increases logistics when applying foundations in the Northern latitudes;

- all-season construction;

- construction time reduction;

- due to the thermal properties of timber, the energy efficiency of the construction built on such foundations in the Arctic regions is increased;

- the upper belt of the structural elements can serve as a floor;

- the use of spatial foundation platform allows to realize the principle of the building closure, which increases the energy efficiency of the first floor;

- use of ventilated space for technical needs.

\section{Results}

\subsection{Expediency of use and scope}

Logistics of high-latitude construction requires development. The use of timber structures can improve logistics due to the transportability of materials. Wood is an environmentally friendly renewable material. A spatial ventilated foundation of timber elements allows to build in a short time due to the structure prefabrication.

The use of reinforced concrete foundations has its significant disadvantages due to the underdeveloped logistics of high-latitude construction and seasonality of installation works. Precast concrete is difficult to transport, and monolithic reinforced concrete significantly slows down the construction process. With all the known advantages of metal construction they contribute to unwanted heat transfer in the thickness of the foundation soil.

Wood has a small coefficient of thermal conductivity, which significantly increases the energy efficiency of the structure as a whole [2-4]. Such material reduces heat transfer from the structure to the footing that is important on the permafrost soils constructed on the first principle. As a result, the risk of thawing of the footing is significantly reduced. Timber structures increase logistics due to their transportability. In addition, due to prefabrication of timber structures, the speed of construction is increased. as discussed by Abovsky.

Platforms can have solutions in the form of: system of cross beams, structural plates, plate-rod structure, as well as in the form of shells and folds. Regardless of the design solution, the spatial foundation platform is prefabricated.

\subsection{Spatial foundation platform in the form of plate-rod structure.}

The spatial foundation platform in the form of a slab-rod structure can be designed from timber slabs made of cross-glued layers of lamellas (CLT) [5-8], forming the upper and 
lower belts of the structure (Fig. 6). Timber elements are made of 2 nd grade larch. Plates in the belts are connected by a hinge. The thickness of the plates of the upper and lower belt can be different thickness depending on the loads. The lattice rods forming the spatial structure are made of bars and arranged obliquely. As a result, the spatial work of the platform is provided and favorable conditions for the transfer of impacts on the footing are created.

According to the set of requirements, the recommended height of the ventilated space of the platform is $1.5 \mathrm{~m}$ [9].

The connection of the plate with the bars can be designed with a support steel platform of a square profile. For fixing the rods of the grid gussets are provided welded to the plate which in turn rests on the support site. To the cobbled brace gussets are connected with the use of steel dowel screws as discussed by Inzhutov.

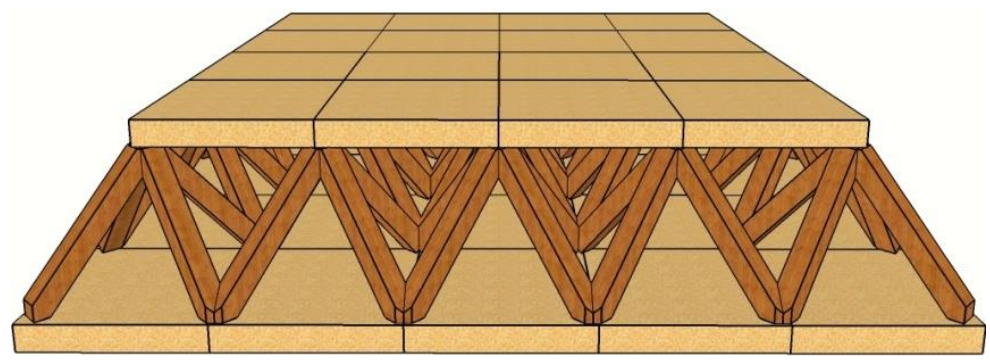

Fig. 6. Spatial foundation platform in the form of a plate-rod structure

\subsection{Spatial foundation platform in the form of a structural plate}

The constructive idea of the foundation is borrowed from the design structures of the covering. A spatial foundation platform in the form of a structural plate is the inverted structural design of the covering (Fig. 7). The structural plate is made of the lower and upper belt of structural elements. Both belts represent a cross-beam system of bars. Between the belts is a system of inclined trusses, elements of which are also presented in the form of bars. It is possible to use laminated veneer timber with large cross-section timber.

In the upper zone of the spatial foundation platform it is advisable to have a heater that fits in the space in the form of a parallelepiped between the bars. In this case, the beams of the upper belt are sheathed with plywood bottom with the top flooring of the first storey.

The joints of the beams in the upper and lower belts can be made on dowels through a steel cross gasket. In turn, the cobbled struts via the gussets and the base platform are connected with steel cross gasket.

According to the set of requirements, the recommended height of the ventilated space of the platform, as in the case of plate-rod structure is 1.5 meters.

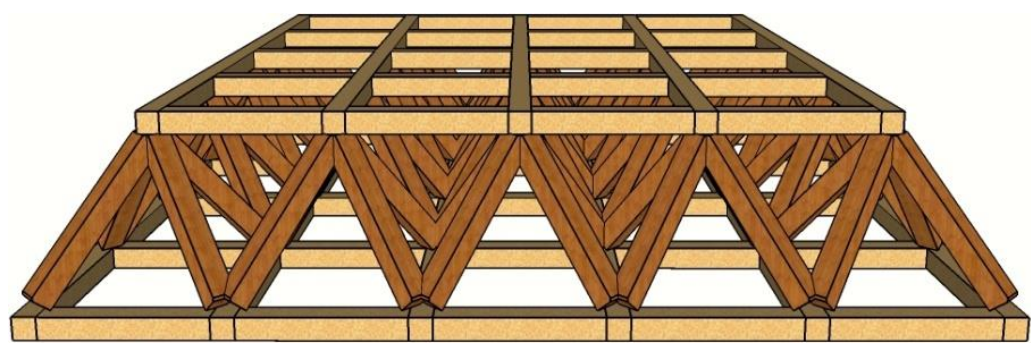

Fig. 7. Spatial foundation platform in the form of a structural plate 


\subsection{Spatial foundation platform in the form of a cross-beam system.}

The spatial foundation platform in the form of a cross-beam system can be represented from CLT panels arranged parallel to the normal and forming a lattice spatial structure (Fig. 8). This type of structural solution of the foundation platform is not necessarily ventilated, but taking into account the spatial work of the structure is applicable in a number of cases.

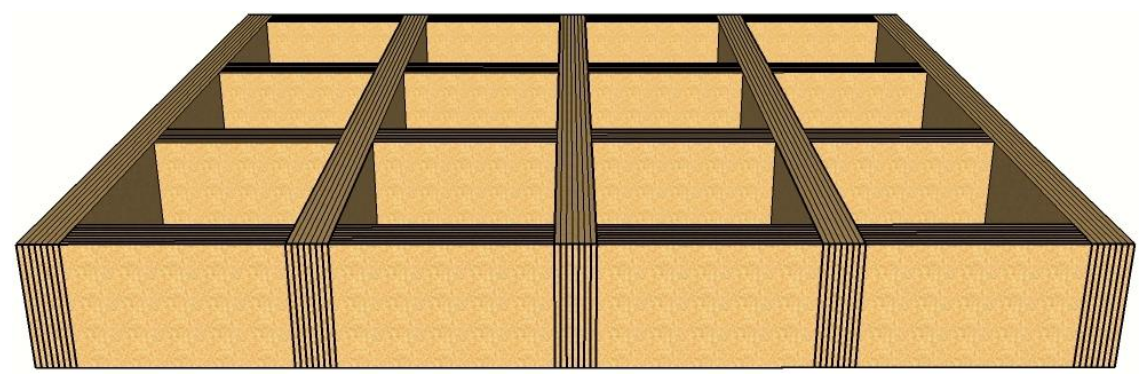

Fig. 8. Spatial foundation platform in the form of a cross-beam system

The space of cells can be filled with soil and insulation. The shape of the foundation cells can be different both as a square, and in the form of a regular hexagon (honeycomb).

Connection of CLT panels can be made on the dowels through the steel cross gasket, glued to the studs with steel plates or pins in the mortise steel gaskets.

\subsection{Spatial foundation platform in the form of folds}

The spatial foundation platform in the form of folds can be made of CLT plates arranged obliquely to each other forming a spatial folded surface of the billowed type (Fig. 9).

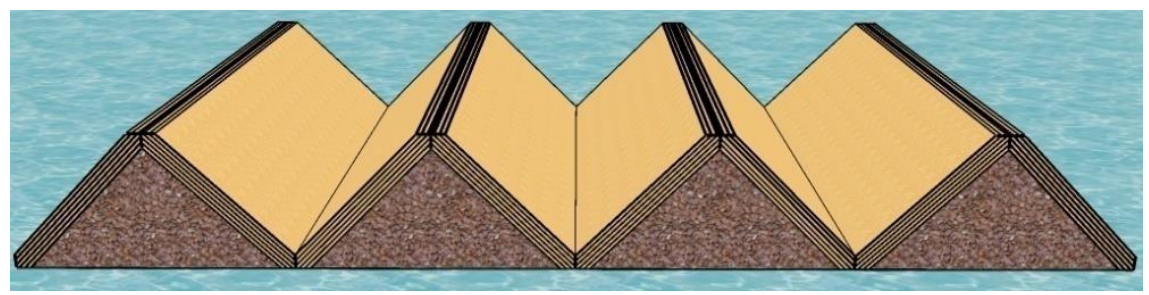

Fig. 9. Spatial foundation platform in the form of the folds of the billowed type.

The space between the CLT plates and the ground of the footing (in the form of a triangular prism) can be filled with low compressible soil and insulation. The space between the CLT plates and the floor of the first storey (in the form of a triangular prism) is ventilated and can be used for the location of communications.

The joints between the CLT plates can be performed using dowel joints and steel plates.

\subsection{Spatial foundation platform in the form of shells}

The spatial foundation platform in the form of shells can be made of CLT plates arranged parallel to the normal, regularly with a certain step (span) (Fig. 10). In the span part a curved thin-walled shell (for example made of plywood) is used under which lies the pillar of the soil massif or prepared base. The space between the curved thin-walled shell and floor of the 1st storey is ventilated and can accommodate networks. 


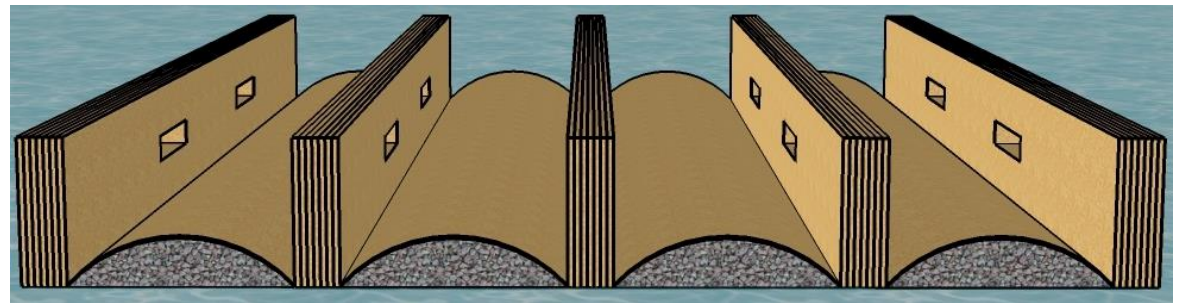

Fig. 10. Spatial foundation platform in the form of a shell with cross-flow.

The curvilinearity of the contact surface of the spatial foundation platform with the base allows to increase the efficiency of the structure during operation on a highly compressible soil footing as discussed by Pronozin [10].

\subsection{Spatial foundation platform with adjustable height}

In the area of heavy snow and wind loads, it is possible to for the spatial foundation platform to be covered with snow, and subsequently for overlying structures, which will adversely affect the performance of the structure. A solution to this problem is the spatial foundation platform of timber items with foundation pillars, the height of which is controlled by a jack device (Fig. 8, 9). Jack devices are located in the zone of positive temperatures, namely in the space of the technical floor. The floor of the technical floor is suspended and when adjusting the height of the floor space remains unchanged. The type of platform with the above technical solution may be different. This technical solution allows to increase the service life of the structure, and reduce the cost of snow removal.

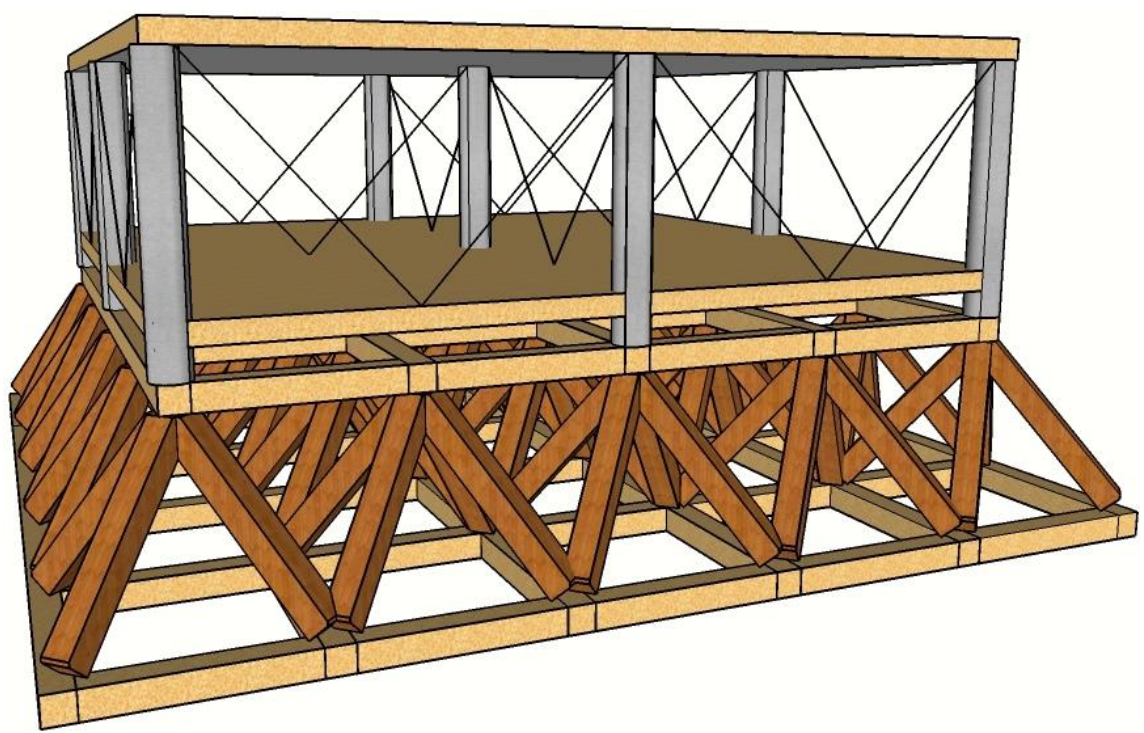

Fig. 8. Spatial foundation platform with adjustable height (lowered). 


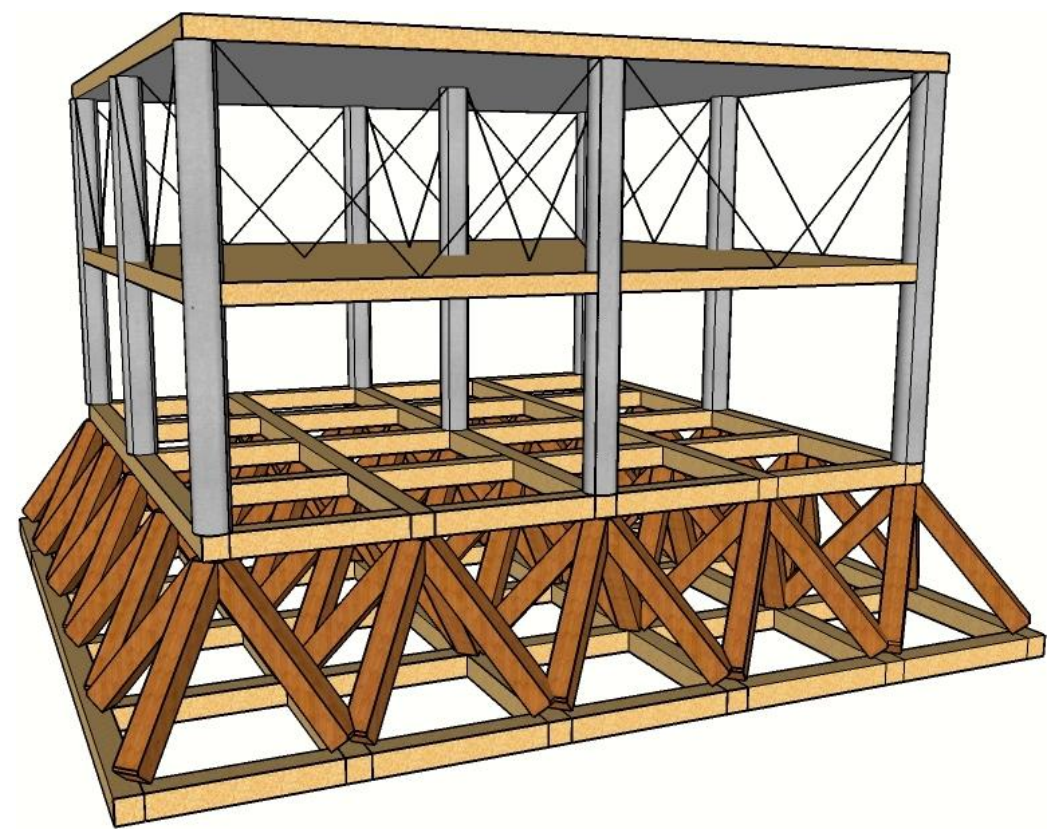

Fig. 9. Spatial foundation platform with adjustable height (raised).

\section{Discussion}

On the basis of the performed comparative analysis it is necessary to highlight the advantages of spatial foundation platforms based on timber in comparison with classical technical solutions. The form of the spatial foundation platform is selected taking into account features of soil of the base and design features of the building. Proven spatial foundations made of reinforced concrete can increase the energy efficiency of the structure by replacing reinforced concrete with wood. Point foundations are appropriate for the construction of temporary buildings and structures, also with small values of loads on the foundations.

\section{Conclusions}

Based on the above types of spatial foundation platforms, it can be concluded that due to the modular design it is possible to obtain the necessary structural solution of the foundation, which due to the thermal characteristics will meet the essential requirements of nordification.

\section{References}

1. N. Khromyshev, V. Ol'shanskii, E. Stolpovskaya, Power Technology and Engineering 2, 19 - 21 (2015) Doi:10.1007/s10749-015-0589-z

2. A.J. Lara-Bocanegra, A. Majano-Majano, F. Arriaga, M. Guaita, Construction and Building Materials 193, 565-575 (2018) doi.org/10.1016/j.conbuildmat.2018.10.224

3. B. Misztal, Springer International Publishing AG 2018 8, 137-163 (2018) doi.org/10.1007/978-3-319-65741-7_8 
4. J. Malesza, C. Miedzialowski, L. Ustinovichius, Journal of Civil Engineering and Management 25(1), 19-26 (2019) doi.org/10.3846/jcem.2019.7738

5. J.W.G. Van De Kuilen, A. Ceccotti, Z. Xia, M. He, Procedia Engineering 14, 16211628 (2011) doi:10.1016/j.proeng.2011.07.204

6. M.F. Laguarda Mallo, O. Espinoza, Journal of Cleaner Production 94, 198-210 (2015) doi.org/10.1016/j.jclepro.2015.01.090

7. K. Jones, J. Stegemann, J. Sykes and Dr. P. Winslow, Construction and Building Materials 125, 690-702 (2016) doi.org/10.1016/j.conbuildmat.2016.08.088

8. Ch. Qian, S. Ménard, D. Bard, J. Negreira, Appl. Sci. 9, 1106 (2019) doi:10.3390/app9061106

9. I. Inzhutov, V. Zhadanov, R. Nazirov, V. Servatinskii, M. Semenov, S. Amelchugov, I. Archipov, Yu. Goncharov, E. Chaikin, IOP Conference Series: Materials Science and Engineering 456, 012046 (2018) doi:10.1088/1757-899X/456/1/012046

10. Z. Ter-Martirosyan, Ya. Pronozin, N. Kiselev, Soil Mechanics and Foundation Engineering 4, 157-164 (2014) doi:10.1007/s11204-014-9272-X 